\title{
Intractable Dysmenorrhea in Adolescent: A Series of Two Cases of Laparoscopic Rudimentary Horn Excision
}

\author{
Reena Yadav, Astha Srivastava \\ Department of Obstetrics \& Gynecology, Lady Harding Medical College and SSKH, New Delhi, India.
}

\section{Corresponding Author: \\ Dr. Astha Srivastava \\ Email: astha1402@gmail.com}

This is an Open Access article distributed under the terms of the Creative Commons Attribution License (creativecommons.org/ licenses/by/3.0).

Received : October 28, 2018

Accepted : December 25, 2018

Published : January 25, 2019

\begin{abstract}
Background: Mullerian anomalies occur in about 3-10\% of females. Women with uterine anomalies are usually asymptomatic unless there is obstruction to menstrual flow, when cyclical pain is experienced. Unicornuate uterus with a non-communicating rudimentary horn with a functioning endometrium is a rare cause of dysmenorrhoea presenting soon after menarche. Case Report: We report two cases of adolescent female presenting with severe dysmenorrhoea. The first patient had unicornuate uterus with a firmly attached noncommunicating rudimentary horn with functional endometrium. Successful laparoscopic excision of rudimentary horn was done. The second case was also similar to the first one but in this case the rudimentary horn was attached to the other horn with a loose band of tissue and in this case the excision was comparatively easier as compared to the first case which was more challenging. Conclusion: Non-communicating rudimentary horn with functional endometrium should be kept as a differential diagnosis in adolescent girls with dysmenorrhoea and ultrasound suggestive of complex adnexal cyst.
\end{abstract}

Keywords: Dysmenorrhoea, Endometrium, Laparoscopy Uterine Anomalies, Uterus.

\section{Introduction}

A unicornuate uterus with a non-communicating rudimentary horn is a rare mullerian anomaly that is most often incidentally found in adults. This condition is often asymptomatic due to the lack of functional endometrium. If a rudimentary uterine horn does not communicate externally but does have an endometrium lined uterine cavity, then clear symptoms of obstructed menstruation may begin soon after menarche and severe dysmenorrhoea will be present.

There are few cases reported with non-communicating rudimentary horn and the functioning endometrium presenting with various complications such as dysmenorrhoea, adenomyosis, ectopic pregnancy and hematometra $[1,2]$. We present two such cases in adolescent girls who presented with intractable dysmenorrhoea.

\section{Case Reports}

\section{Case 1}

A 13-year-old unmarried girl presented to gynaecology out-patient department with severe dysmenorrhoea. Patient had attained menarche five months prior to presenting to our hospital. Her initial cycles were regular with average flow without associated dysmenorrhoea. Four months after menarche she started to have severe dysmenorrhoea lasting for 4-5 days. The pain was extremely severe and was relieved only with injectable analgesics. There was no significant past and family history. On examination patient was moderately built and nourished. Her general physical and per-abdomen examination was normal. Abdominal ultrasound showed a thick walled complex cystic area of size $1.6 \times 1.6 \times 1.4 \mathrm{~cm}$ closely abutting the right ovary. Myometrium showed normal homogenous 
echotexture and endometrial thickness (ET) of 4.9 $\mathrm{mm}$. Impression of abdominal ultrasound was right adnexal complex cyst/endometriotic cyst. Her MRI (sections being taken in axial, saggital and coronal planes) findings was suggestive of bicornuate uterus with right lateral half of the cavity distended with hemorrhagic contents [Fig.1]. Per-operatively uterus was enlarged with wide fundus and dimple in it suggestive of two horns with right horn being more enlarged compared to left [Fig.2]. The right non-communicating horn was firmly attached to the left communicating horn of uterus. Both tubes and ovaries were normal looking. Area between the two horns was caught with laparoscopic forceps [Fig.3] and incision was started with harmonic scalpel with precision more towards right [Fig.4]. During this step while going deep the cavity got opened (around $3 \mathrm{~cm}$ ) which was filled with dark altered blood. Rest of the horn was excised by detaching it from lateral attachments of round ligament, utero-ovarian and uterine artery. Uterine artery was on right infero-lateral aspect which was coagulated. Raw area was sutured. Excised horn was put in endobag and removed through one of the port. Histopathological report of the specimen confirmed uterine wall lined by endometrium.

\section{Case 2}

A 18-year-old unmarried girl presented to our gynaecology out-patient department with severe

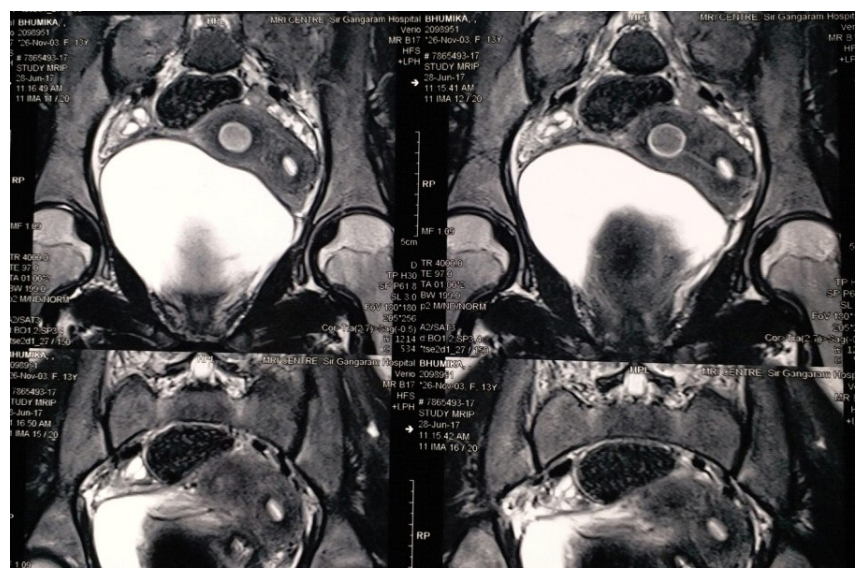

Fig.1: MRI film suggestive of bicornuate uterus.

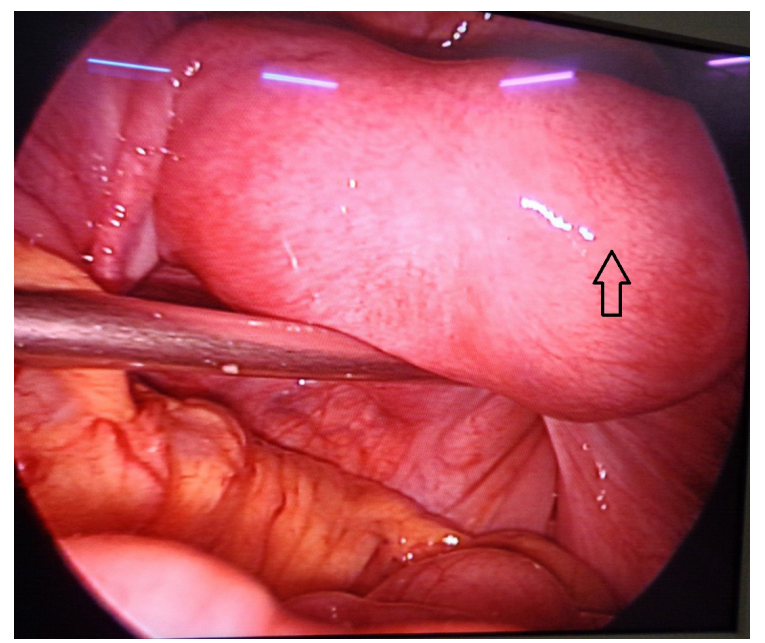

Fig.2: Rudimentary horn (right side) filled with blood (arrow).

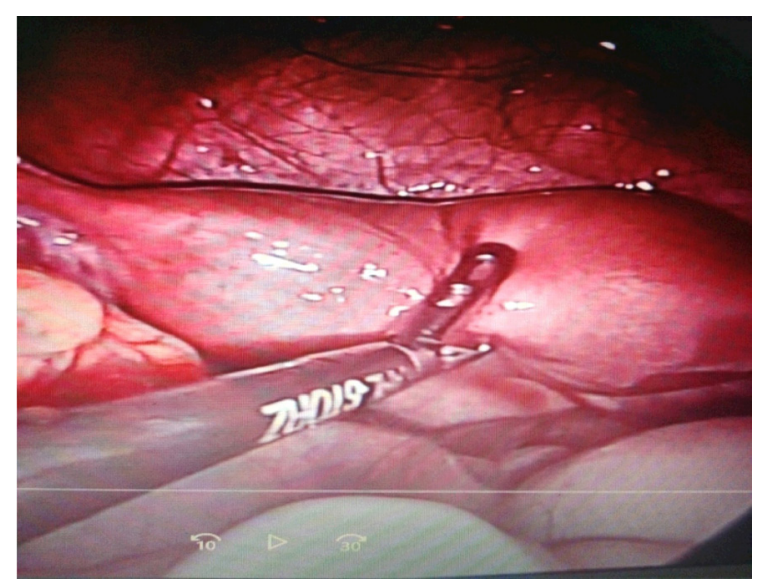

Fig.3: Depression between the two horns held with laparoscopic forceps.

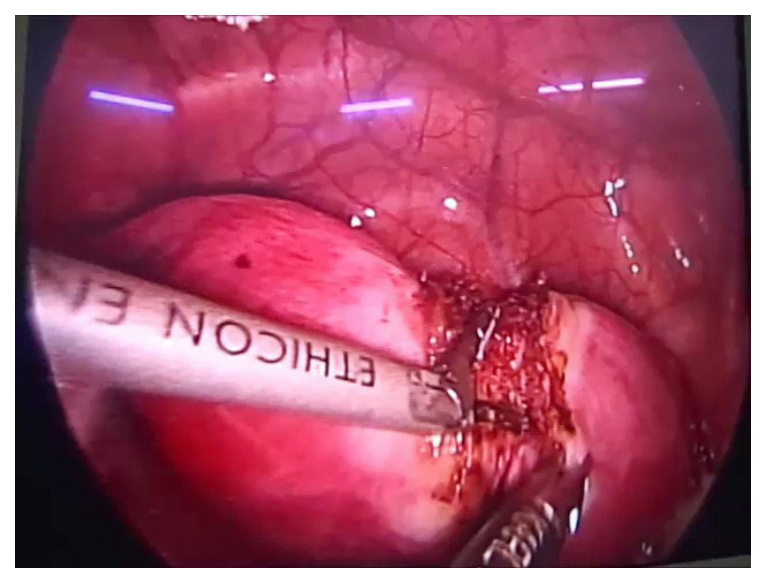

Fig.4: Excision of rudimentary horn with harmonic scalpel. 
dysmenorrhoea relieved only by injectable analgesics. The menstrual history was similar to the first case with gradually progressive dysmenorrhoea which started about six months after menarche. Contrast enhanced computed tomography was suggestive of bicornuate uterus with functioning and obstructed non-communicating rudimentary horn. Patient was planned for laparoscopy. Peroperatively there was a right rudimentary horn [Fig.5]. Bilateral fallopian tubes and ovaries were normal looking. There was a loose band of tissue between the two horns. Right sided round ligament was clamped and coagulated followed by right cornufundal structures followed by right uterine artery. The midline band of tissue between the two horns was coagulated and cut [Fig.6]. The right rudimentary horn was then removed in endobag.

\section{Discussion}

A unicornuate uterus with a non-communicating rudimentary horn occurs because of defect in lateral fusion of the mullerian ducts as per American Fertility Society Classification of uterovaginal anomalies. Rudimentary horns may or may not have a functioning endometrium. Associated medical problems include dysmenorrhoea, infertility, endometriosis, and ectopic pregnancy. There may be ipsilateral renal agenesis. In the presence of active endometrium retrograde menstruation may lead to the development of endometriosis. The incidence of congenital uterine anomaliesin fertile population is $1 / 200$ to $1 / 600$. The incidence of rudimentary horn is rare $(1: 100,000)$. In $80-90 \%$ cases there is no communication with other horn. Rudimentary horn could be either firmly attached to the unicornuate uterus as in our first patient or separated by a loose band of tissue as was there in second case.

Obstructive uterine anomalies usually present with chronic cyclic pain, with increasing severity of dysmenorrhoea with each subsequent menstrual cycle. Symptoms may be severe enough to interfere with quality of life and pain may not

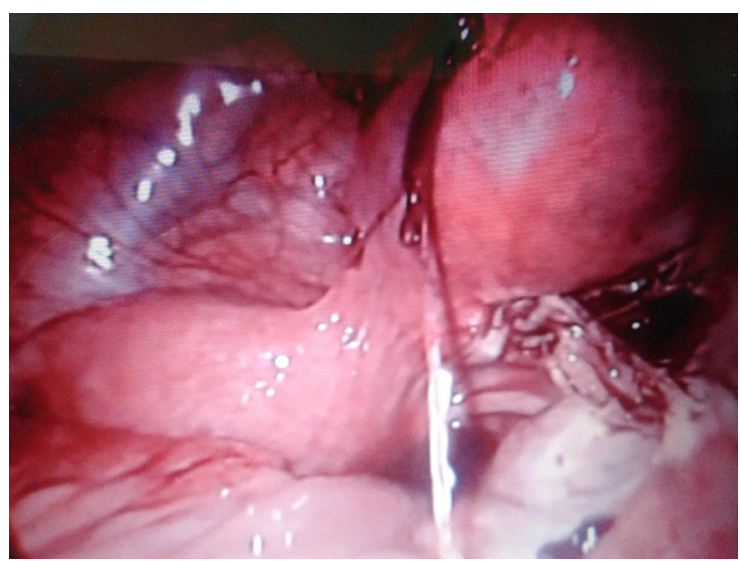

Fig.5: Excision of the midline septum between right rudimentary horn and normal horn.

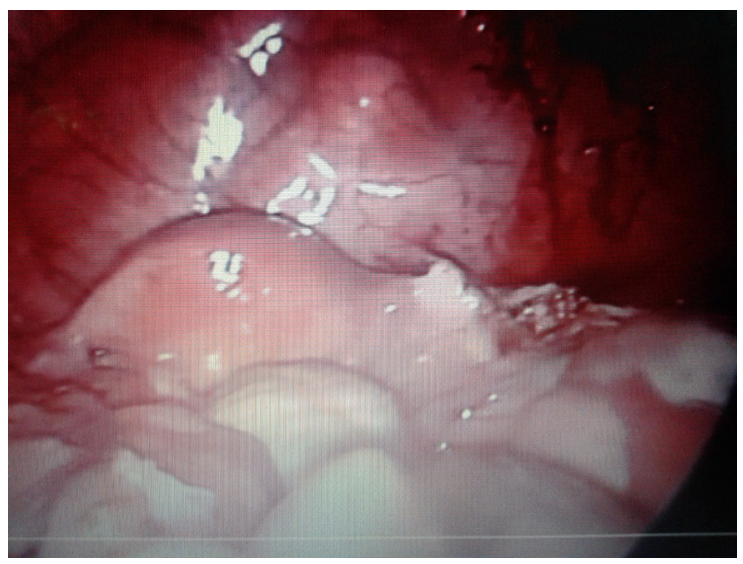

Fig.6: Remaining left uterine horn after excision of the right horn.

respond to usual analgesics as was there in our cases. 3-dimensional or 4-dimensional ultrasound or magnetic resonance imaging is used for diagnosis of mullerian anomalies. [3]. Laparoscopy is necessary for a definitive diagnosis and for removal of the abnormal uterine horn. Most of the information on unicornuate uterus with rudimentary horn has been gathered from case reports such as these two cases. In both these cases, a laparoscopic approach was used to excise the rudimentary horn. Similar case of an adolescent girl with functional rudimentary horn has been reported by Anjali et al. [4] where rudimentary horn was removed by laparotomy. In adults, the uterine horn tends to be small, nonfunctional, and incidentally discovered. In our first 
case a large horn filled with old blood was present which was firmly attached to the uterus. It was challenging to remove such big firmly attached horn through laparoscopy. A high index of suspicion for Mullerian duct abnormality is therefore needed in teenagers with dysmenorrhoea to prevent delay in diagnosis [5].

\section{Conclusion}

Non-communicating rudimentary horn with functional endometrium should be kept as a differential diagnosis, in adolescent girls with dysmenorrhoea and ultrasound suggestive of complex adnexal cyst. Patient should be investigated accordingly. These patients should be managed by expert surgeon preferably by laparoscopic approach.

Contributors: RY: literature search, manuscript editing, patient care; AS: literature search, manuscript preparation, patient care. AS will act as guarantor of this study. Both authors approved the final version of this manuscript. Funding: None; Competing interests: None stated.

\section{References}

1. Acién P, Bataller A, Fernández F, Acién MI, Rodríguez JM, Mayol MJ. New cases of accessory and cavitated uterine masses (ACUM): a significant causeof severe dysmenorrhea and recurrent pelvic pain in young women. Hum Reprod. 2012;27:683-694.

2. Arab M, Mehdighalb S, Khosravi D. Functional rudimentary horn as a rare cause of pelvic pain: a case report. Iran Red Crescent Med J. 2014;16:e19351.

3. Spitzer RF, Kives S, Allen LM. Case series of laparoscopically resected non communicating functional uterine horns. J Pediatr Adolesc Gynecol. 2009;22:E23-E28.

4. Rani A, Kumari M, Shipra. A case of non-communicating uterine horn containing functional endometrium. Gynecol Obstet (Sunnyvale). 2015;5:320.

5. Badawy SZA, Montgomery S. Severe primary dysmenorrhoea due to functioning non communicatimg rudimentary horn. J Gynecol Surg. 2009;25:23-27. 